\title{
SELF-ADJOINT BOUNDARY CONDITIONS AND INTERLACING OF EIGENVALUES FOR THE STURM-LIOUVILLE EQUATION ON GRAPHS
}

\author{
SONJA CURRIE
}

Abstract. Applying the approach of Kostrykin and Schrader, [14], an explicit characterisation of self-adjoint boundary conditions at the nodes or vertices of a graph for the Sturm-Liouville equation is given. This is then proven to be equivalent to the conditions for self-adjointness of the corresponding system boundary value problem with separated boundary conditions. In addition, using an example, it is shown that the complete graph configuration is incorporated in the system boundary condition at the terminal end point. Making use of the separated system formulation, via matrix Prüfer angle techniques, an interlacing property of the eigenvalues for a self-adjoint Sturm-Liouville boundary value problem on a graph is ascertained.

Mathematics subject classification (2010): 34B45, 34L05, 34B24, 34L15. interlacing.

Keywords and phrases: Self-adjoint, differential operators on graphs, eigenvalues, Sturm-Liouville,

\section{REFERENCES}

[1] S. Agmon, Lectures on elliptic boundary value problems, Princeton, 1965.

[2] F. Ali Mehmeti, A characterization of a generalized $C^{\infty}$-notion on nets, Integral Equations and Operator Theory 9 (1986), 753-766.

[3] J. Avron, Adiabatic quantum transport in multiply connected systems, Reviews of Modern Physics. 60, 4 (1988), 873-915.

[4] R. CARLson, Adjoint and self-adjoint differential operators on graphs, Electronic J. Differential Equations 1998, 6 (1998), 1-10.

[5] E. A. Coddington, N. Levinson, Theory of ordinary differential equations, McGraw-Hill, New York, 1955.

[6] S. Currie, B. A. WATson, Eigenvalue asymptotics for differential operators on graphs, J. Com. Appl. Math. 182 (2005), 13-31.

[7] S. Currie, B. A. Watson, it $M$-matrix asymptotics for Sturm-Liouville problems on graphs, J. Com. Appl. Math. 218 (2008), 568-578.

[8] S. CurRie, B. A. WAtson, The M-matrix inverse problem for the Sturm-Liouville equation on graphs, Proc. Royal Soc. of Edinburgh. 139A (2009), 775-796.

[9] G. J. Etgen, Two Point Boundary Problems for Second Order Matrix Differential Systems, Transactions of the American Mathematical Society 149 (1970), 119-132.

[10] P. EXNER, M. Helm, P. STOLLMANn, Localization on a quantum graph with a random potential on the edges, Rev. Math. Phys. 19 (2007), 923-939.

[11] P. EXNER, P. ŠEBA, P. ŠŤOVIČEK, Quantum interference on graphs controlled by an external electric field, J. Phys. A 21, 21 (1988), 4009-4019.

[12] N. GerasimenKo, B. PAVlov, Scattering problems on non-compact graphs, Theoretical and Mathematical Physics 74, 3 (1988), 230-240.

[13] M. HARMER, Hermitian symplectic geometry and extension theory, J. Phys. A: Math. Gen. 33 (2000), 9193-9203.

[14] V. Kostry Kin, R. Schrader, Kirchhoff's rule for quantum wires, J. Phys. A: Math. Gen. 32 (1999), $595-630$. 
[15] V. Kostrykin, R. Schrader, Quantum wires with magnetic fluxes, Commun. Math. Phys. 237 (2003), 161-179.

[16] T. Kottos, U. Smilansky, Chaotic scattering on graphs, Phys. Rev. Lett. 85 (2000), 968-971.

[17] P. Kuchment, Graph models for waves in thin structures, Waves Random Media 12 (2002), R1-R24.

[18] P. Kuchment, Differential and psuedo-differential operators on graphs as models of mesoscopic systems, Analysis and Applications, eds. H. Begehr, R. Gilbert, M. W. Wang (Dordrecht: Kluwer Academic), 2003, 7-30.

[19] M. A. Naimark, Linear Differential Operators, Part I, Frederick Ungar Publishing co., New York, 1967.

[20] Yu. V. Pokornyi, V. L. Pryadiev, Some problems of the qualitative Sturm-Liouville theory on a spatial network, Russian Math. Surveys 59, 3 (2004), 515-552.

[21] R. E. Showalter, Hilbert Space Methods for Partial Differential Equations, EJDE Monographs, 2001.

[22] J. VON BELOW, A characteristic equation associated to an eigenvalue problem on $c^{2}$-networks, Linear Algebra and Applications 71 (1985), 309-325.

[23] V. YURKO, Inverse spectral problems for the matrix Sturm-Liouville equation on a finite interval, Inverse Problems 22 (2006), 1139-1149. 